\title{
Ética del deporte \\ La propuesta sustancialista de Lumpkin, Stoll y Beller, desde el procedimentalismo ético
}

\author{
RAÚl FRANCISCO SEBASTIÁN SOLANES \\ Universidad de Valencia (España) \\ Raul.Sebastian@uv.es
}

\begin{abstract}
Resumen
En este artículo presento de forma crítica la propuesta de ética del deporte como «bolsa de virtudes» que elaboran tres autores estadounidenses que son Lumpkin, Stoll y Beller. Dicha propuesta debe entenderse como una manifestación de sustancialismo neoaristotélico en deporte, con una clara influencia de MacIntyre. Mi objetivo es mostrar cómo dicho sustancialismo no es suficiente para una propuesta ética del deporte, por lo que abogo por incorporar algunos de los presupuestos del procedimentalismo ético aplicado al deporte.
\end{abstract}

Palabras claves: ética del deporte, virtudes, sustancialismo neoaristotélico, procedimentalismo.

\section{Sport Ethics \\ The substantialist proposal of Lumpkin, Stoll and Beller, from ethical proceduralism}

\begin{abstract}
In this article the author presents critically the proposed sports ethics as «bag of virtues» that Americans made three authors are Lumpkin, Stoll and Beller. This proposal should be seen as a manifestation of substantialism neoaristhotelic in sport, with a clear influence of MacIntyre. My aim is to show how such substantialism not enough for a sports ethics proposal therefore advocate incorporate some of the ethical proceduralism budgets applied to sport.
\end{abstract}

Key words: ethic of sport, virtues, sustancialism neo-aristhotelic, proceduralism.

Doctor Europeo en Sociología por la Universidad de Bolonia. Doctorando en Filosofía en la Universidad de Valencia con la dirección de Adela Cortina. Entre sus últimas publicaciones cabe destacar: "Sociologia dello sport: Lo stato della questione" (2012), "The ethical problem of intimidation in sport: From the violence to the responsibility" (2012), "Ética del deporte y Personalismo In-sistencial: Ismael Quiles nuevas aportaciones" (2012), "Horizonte y necesidad de una economía ética en deporte" (2012), y "Deporte y migración: Las diferencias sociológicas de género en deporte, desde la ética” (2012). 


\section{E1 horizonte filosófico de la virtud: De Homero a MacIntyre}

La ética de las virtudes resurge en el panorama actual de la filosofía con el principal respaldo de pensadores como Anscombe, y sobre todo, MacIntyre, que es quien más influye en las nuevas propuestas de ética del deporte. Con el fin de aproximarnos a la propuesta de ética del deporte como ética de la virtud, la cual me propongo exponer en el presente texto, creo conveniente ver muy brevemente lo que ha supuesto el término virtud a lo largo de la historia de la filosofía. Para este propósito me remontaré a la época heroica hasta llegar a MacIntyre para comprobar si, efectivamente, la propuesta de los nuevos éticos del deporte puede considerarse una ética del deporte como ética de la virtud.

La historia del término filosófico de virtud es mucho más antigua y se remonta a los tiempos heroicos de la Ilíada y la Odisea de Homero. En el contexto homérico, la virtud o «areté» era propia de los héroes, dado que la situación social era el principal determinante de los valores o virtudes y solo los héroes, por su linaje y riqueza, podían ser virtuosos, nunca los simples sirvientes o campesinos. Los valores o virtudes de la sociedad homérica eran dados, predeterminados por el puesto del hombre en la sociedad y dependían de la generosidad azarosa del dios y del destino. Moral y estructura social son una y la misma cosa en la sociedad que describe Homero. De este modo la virtud en la época heroica está vinculada a lo socialmente singular y local, por lo que toda pretensión ilustrada de universalidad sería una mera ilusión y sólo se puede poseer una virtud como parte de una tradición dentro de la cual la heredamos y la discernimos de una serie de predecesores.

En la época clásica se produce un cambio en la concepción de las virtudes cuando la comunidad moral primaria ya no es el grupo de parentesco, sino la ciudad-estado, no en sentido general, sino la democracia ateniense en particular. En este caso la cooperación es la característica esencial de las virtudes de los atenienses como puede verse en el «Discurso fúnebre» de Pericles que se recoge en la Historia de la guerra del Peloponeso de Tucídides, donde el célebre político de Atenas atribuye a sus conciudadanos la incesante actividad en la persecución de los propios fines, el afán por hacer y extenderse más y la moderación en las maneras. En el caso del pensamiento filosófico de Platón, el concepto de virtud sigue siendo un concepto político, dado que la descripción platónica del hombre virtuoso es inseparable de su descripción de ciudadano político.

Será Aristóteles quien más hable de la virtud en sus escritos sobre ética, principalmente en la Ética a Nicómaco. Debemos al Estagirita una importante reflexión en torno a la virtud y su vinculación a la ética que 
va a marcar para siempre su estudio y aplicación. En la Ética a Nicómaco (II, 1, 1103a) Aristóteles distingue entre dos tipos de virtud, las virtudes dianoéticas, que deben su origen e incremento a la enseñanza y por ello requieren de experiencia y tiempo, y las virtudes éticas, que proceden de la costumbre y no se producen en nosotros por naturaleza, ya que nada que proceda de la naturaleza se modifica por la costumbre. Por esta razón las virtudes no se producen ni por naturaleza ni contra natura, sino por tener una aptitud natural para recibirlas y perfeccionarlas mediante la costumbre. La virtud de un hombre será el hábito por el cual éste se hace bueno y por el cual se ejecuta bien su función propia dado que la virtud ética tiene que ver con las pasiones y las acciones. En ellas se pueden dar el exceso o el defecto, pero también la recta razón que es el término medio entre el exceso y el defecto. De modo que para Aristóteles la virtud debe consistir en un hábito selectivo basado en el término medio entre el exceso y el defecto.

Ahora bien, en la ética de las virtudes aristotélica, juega un papel muy importante tanto el deseo como la elección y la deliberación, pues son ellas quienes ponen en funcionamiento la acción del sujeto moral para perseguir un fin, que será la felicidad. El objeto de la elección y de la deliberación será lo mismo, por ello afirmará que son los medios para el fin, es decir, las acciones relativas a éstos serán conformes a la elección y voluntarios, por lo que reside en los sujetos morales la capacidad de deseo, deliberación y elección tanto de la virtud como del vicio. Obviamente si elegimos el vicio nos alejaremos del fin natural al que tiende todo el hombre como es la felicidad, entendida como actividad contemplativa de lo divino, pero si por el contrario elegimos la virtud conseguiremos alcanzar dicho fin. Para Aristóteles hay tres cosas que vienen a regir la acción y la verdad, que son la sensación, el entendimiento y el deseo. La sensación no es principio de acción, como sí lo es el deseo, ya que la virtud moral es la disposición relativa a la elección, y ésta última es un deseo deliberado. Por este motivo queda constituido que el principio de la acción es la elección y que ésta no es más que un deseo deliberado. Es por ello que la definición de hombre se establece como inteligencia deseosa o deseo inteligente, pues el deseo es quien pone en funcionamiento la deliberación y ésta la elección de aquellos medios, en nuestro caso las virtudes, que nos deben acercar al fin (Ética a Nicómaco, VI, 2, 1139b).

También Aristóteles establece un listado de virtudes éticas como son el arte, la ciencia, la prudencia, la sabiduría y el intelecto, aunque a mí me interesa especialmente la virtud práctica de la prudencia, pues en ella se cimentan todas las demás y el comportamiento mismo del hombre virtuoso. El hombre prudente es aquel capaz de discernir bien entre lo 
que es justo e injusto, en términos generales el prudente es hombre reflexivo capaz de razonar bien en vistas de un fin bueno aplicándose a la buena deliberación y a la buena elección de los medios que nos conducen al fin natural, esto es, la felicidad. Pero la prudencia debe entenderse como virtud práctica que se refiere a lo más particular, es decir, a como obrar en cada caso particular. Porque el hombre prudente es aquel que sabe cómo actuar en el caso particular, en consecuencia el hombre virtuoso debe ser concebido como la personificación de la prudencia en el caso particular, tal y como aclara acertadamente Michelakis (1961: 7) siguiendo los presupuestos de la moral aristotélica. Así, la misma prudencia implica un doble acto cognitivo, pues no sólo se refiere al conocimiento de los fines, sino también al de los medios que nos acerca al fin (Moncho, 1972: 93). De este modo, el hombre llevará a cabo su obra mediante la prudencia y la virtud moral, pues la virtud hace recto el fin propuesto y la prudencia los medios que a él conducen (Ética a Nicómaco, VI, 12, 1144a).

En la línea de la tradición aristotélica destaca en nuestro tiempo la concepción de virtud que establece Alasdair MacIntyre en After virtue, pues será a partir de esta obra cuando se forjan las nuevas propuestas de ética del deporte. MacIntyre supone una recuperación de una concepción, de carácter marcadamente neoaristotélico, de ética de la virtud. En dicha propuesta se dispone a devolver en el panorama actual de la ética, nociones, como es la de virtud, que ya habían sido abordadas sobradamente en época heroica, clásica y medieval, y que posteriormente fueron abandonadas o desplazadas por las éticas aparecidas en época moderna e ilustrada. La Modernidad había supuesto una línea divisoria con el pensamiento clásico. En efecto, mientras que los clásicos piensan en términos de virtud englobante donde no se separa la consideración de lo justo y lo bueno; en cambio, los contemporáneos sí establecen una separación entre lo bueno y lo justo, siendo esto último el límite negativo de las peripecias éticas individuales (Moncho, 2002: 155-156).

MacIntyre (2001: 233) establece un desarrollo lógico del concepto de virtud que divide en tres fases: la primera exige una descripción de lo que llama práctica junto con los bienes internos y externos, junto con los modelos de excelencia que se siguen de los anteriores y la observancia a reglas; la segunda la que llama como orden narrativo de una vida humana; y en tercer lugar la descripción de lo que es una tradición moral. La definición provisional de virtud es la de una cualidad humana adquirida cuya posesión y ejercicio tiende a hacernos capaces de lograr los bienes internos a la práctica. Las virtudes que nos acercan a este propósito son las de valor, justicia y honestidad, que son los componentes necesarios de toda práctica que contenga bienes internos y 
modelos de excelencia. No cumplir con lo que establecen estas tres virtudes supone cometer trampas en busca de bienes externos, de modo similar al niño del ejemplo del juego de ajedrez que rompe las reglas del juego en vistas de un bien externo. Las virtudes son además los bienes por referencia a los cuales definimos nuestra relación con los demás quienes también comparten los propósitos éticos que implica una práctica virtuosa (MacIntyre, 2001: 238).

\section{Valor y razonamiento moral en el deporte en A. Lumpkin, S. K. Stoll Y J. M. Beller}

El deporte moderno presenta unas características diferentes a los juegos rituales del mundo homérico, e incluso diferente a la época clásica de Platón y Aristóteles. El deporte moderno debe entenderse como un producto de la sociedad industrial, lo que marca algunos de los puntos que le caracteriza, como son el secularismo, la igualdad de oportunidades, la especialización, la racionalización, la burocratización, el espectáculo y la cuantificación basada en el mayor rendimiento (Guttmann, 1978). Pero además, el deporte moderno surge dentro de un contexto donde aparecen términos como el de wellness, fitness o leisure, que han irrumpido y han consolidado una forma de vida, una revolución no silenciosa que se ofrece como signo de los tiempos (Russo, 2011: 13). Dichos términos se ven favorecidos con la aparición del sistema deportivo como uno de los escenarios principales de nuestras sociedades, convirtiéndose en el ejemplo paradigmático del hombre performance. El deporte moderno se convierte en un aparato burocrático que se preocupa por formar deportistas especializados que se centren única y exclusivamente en su vertiente deportiva para ser el mejor en su modalidad (Guttmann, 2000).

Todo lo anterior ha producido la aparición de comportamientos antideportivos que requieren de una respuesta por parte de la ética, lo que ha tenido como consecuencia la aparición de corrientes y pensadores que elaboran sus propuestas de ética del deporte como la que abordamos en este apartado. Una de esas corrientes es la propuesta de ética del deporte como «bolsa de valores morales» que ofrecen Brenda Bredemeir y David Shields en su libro Character Development and Physical Activity, o Ángela Lumpkin, Sharon K. Stoll y Jennifer. M. Beller en su libro colectivo Sport ethics: applications for Fair Play en la que me centraré. La primera propuesta debe entenderse como una forma de sustancialismo neoaristotélico con una clara pretensión de acercarse a la propuesta de MacIntyre y aplicarla a la práctica deportiva. Recordemos que el 
sustancialismo ético, que contaría con dos grandes corrientes como es el neohegeliano y el neoaristotélico, se caracterizaría por obtener una concepción de la moral que no es representativa por sus normas, que ocupan un lugar secundario y subordinado. En el primer plano de reflexión ética, deben situarse los bienes y las virtudes, que desplazan a un lugar secundario $y$ subordinado a las normas $y$ deberes universalmente justificables. Lo bueno prima por encima de lo correcto y lo correcto siempre es establecido desde lo bueno. Lo bueno es aquello que beneficia a la comunidad entera y no solo a una parte (Cortina, 2001: 80-81).

Por su parte, la propuesta de Lumpkin, Stoll y Beller, debemos situarla en el momento de transición en que se incrementa el interés por la ética del deporte, desde el enfoque de una ética de la virtud. Evidenciando un progresivo alejamiento de los planteamientos que reducían la reflexión ética en deporte a meros códigos éticos o de buena conducta que se habían convertido en los modelos imperantes en ese momento. Esto había provocado que el deontologismo basado en estos códigos fuera la corriente o, mejor dicho, la tendencia imperante en el debate relacionado a la ética del deporte, abogando a favor de una propuesta de ética del deporte como ética de la virtud y en donde la influencia de MacIntyre y de su obra After virtue es clave.

Lumpkin, Stoll y Beller, centran su atención en el deporte y las instituciones deportivas modernas de EEUU. Entienden que el principal problema al que se enfrenta el deporte estadounidense es el enmascaramiento inconsciente del razonamiento moral en las prácticas deportivas. Esta falta de atención se debe a que los deportistas no aprenden a pensar y reflexionar acerca de las cuestiones e implicaciones éticas de su práctica. Dedicándose tan sólo a seguir ciega y pasivamente las reglas del deporte y generando una práctica que en la mayoría de ocasiones dista mucho de un comportamiento ético y moral.

Para construir una ética del deporte como ética de la virtud que incorpore la riqueza del pensamiento neoaristotélico de MacIntyre, deben establecerse al menos tres fases del desarrollo lógico de virtud con el fin de comprender cuál es su alcance en el caso de la práctica deportiva. La primera de ellas debería ser la descripción de la práctica que implicaría ver qué bienes internos son característicos de ella, viendo qué modelo de excelencia se persigue y qué reglas morales debemos aceptar. La segunda fase tendría que ver con el orden narrativo de una vida humana. Para terminar, deberíamos recurrir a lo que es un concepto de tradición moral, viendo en las prácticas contemporáneas las prácticas de todos aquellos que nos han precedido (MacIntyre, 2001: 233). 
De entrada no encontramos en estos autores una definición exacta de lo que es una práctica como sí la encontramos en MacIntyre, como cualquier forma coherente y compleja de actividad humana socialmente establecida, que posee unos bienes internos a través de los cuales alcanzamos los modelos de excelencia y que tiene que ver con la observancia a una reglas, que pese a su lugar secundario y subordinado, deben acompañar a toda práctica si quiere ser virtuosa. En el caso del deporte, las normas y reglas ya vienen dadas, siempre bajo la supervisión del Comité Olímpico Internacional, no se consensuan cada vez por sus afectados en el momento de iniciar el encuentro deportivo. Ahora bien, cimentar una propuesta de ética del deporte, tal y como hacen Lumpkin, Stoll y Beller, en un conjunto de virtudes pre-establecidas puede inducirnos a pensar que recurrimos al argumento de autoridad, al peso de una tradición de donde emanan dichas virtudes. Esto nos llevaría a ver cómo arbitrar las discrepancias que surgen en las diferentes tradiciones históricas, además de los distintos contextos sociales, asumiendo que el deporte es una práctica que se desarrolla en las distintas culturas. De hecho, Butcher y Schneider (2002: 24) establecen una dura crítica a la concepción de Lumpkin, Stoll y Beller, pues definen la ética del deporte o el fair play como «bolsa de virtudes». Ellos entienden que esta propuesta debe ser rechazada porque no ofrece un método defendible para decidir qué características o acciones deben caer en las definiciones relevantes, así como tampoco ofrece un método para arbitrar entre pretensiones enfrentadas.

Sin embargo, sí encontramos en estos autores la definición de lo que debe ser un razonamiento moral referido al deporte y que nos conducirá a su noción de valor y virtud. El razonamiento moral tiene que ser lógico y coherente, además de imparcial. Esto se debe a que la lógica viene a ser la esencia del razonamiento moral, pero también debe ser coherente, lo que implica tener en cuenta el pasado, el presente y futuro de nuestras decisiones. $\mathrm{Y}$, finalmente, imparcial, entendiendo ésta como una forma de justicia de nuestro razonamiento moral y de su posterior aplicación en el terreno deportivo.

Decir que toda práctica y todo razonamiento moral en torno a ésta implicaría tener en cuenta el pasado, presente y futuro de dicha práctica, indica que ésta se inserta en una tradición. No sólo se refiere a los bienes internos o conlleva modelos de excelencia y obediencia a reglas, sino que entrar en una práctica supone aceptar la autoridad de estos modelos y la cortedad de nuestra acción. Ello se debe a que toda práctica tiene una historia y proviene de una determinada tradición. Entendiendo por tradición como aquellos datos previos a nuestras vidas, que le confieren su propia particularidad y que hemos heredado, formando parte de la 
misma (MacIntyre, 2001: 273). Pero también implicaría la imparcialidad, puesto que la pretendida cooperación en la práctica deportiva, junto al reconocimiento de la autoridad de los modelos de excelencia, bienes internos, obediencia a reglas dentro del deporte y los correspondientes méritos, exigen la imparcialidad a la hora de juzgarse a uno mismo y a los demás. Las virtudes constituirán los bienes por referencia a los cuales definimos nuestra relación con los demás. En efecto, que nuestro razonamiento sea imparcial en deporte equivale a decir que tiene que ser justo o equitativo y legítimo, haciendo que nuestro comportamiento esté libre de prejuicios, de fraude y de injusticia.

Lumpkin, Stoll y Beller (1994: 13) entienden que el razonamiento moral es una forma crítica, que no criticona, de hacer preguntas y respuestas cuyo fin y propósito es descubrir la verdad moral que en el caso del deporte nos ayudaría a concretar cuál es el fin del deporte, viendo el importante papel que desempeñan los valores morales, para conseguir dicho fin y no errar el camino. Ante la pluralidad de situaciones que acontecen en el transcurso de la competición deportiva encontramos una multitud de razonamientos ad hoc que intentan dar una salida racional a la forma de comportarse en cada situación. Pero no todas las situaciones y actuaciones que surgen en el deporte pueden ser catalogadas como prototipo de razonamiento moral. Ello se debe a que muchas veces el razonamiento que se hace para un tipo de actuación estratégica en el campo deportivo y dista mucho de una consideración moral como muestra según estos autores el caso del entrenador Paul Brown.

El entrenador Brown inició su carrera en el Instituto Massillon de Ohio. En el año 1928 se dio cuenta de que en el equipo de fútbol americano que él entrenaba, los runnig back, eran muy lentos en reaccionar y preparar la ofensiva desde el backfield. Brown revisó las normas de este deporte para obtener una ventaja a su favor y se percató de que según la normativa oficial no existe ninguna mención a lo que podría o debería tener el uniforme que utilizan los jugadores, salvo que deben llevar un número en la espalda. Brown utilizó esta circunstancia a su favor y vio en ella una oportunidad que podría beneficiarle en los entrenamientos. Sin pensarlo dos veces cortó un balón en dos mitades y las colocó en los hombros de los jugadores que ocupaban la posición de backfield, logrando que estos corredores obtuvieran más ventaja y desempeñaran su cargo con mayor precisión.

En efecto, no podemos decir que el razonamiento de Brown aplicado a la situación concreta que le tocó vivir deje de ser un razonamiento. De hecho, es un ejemplo prototípico de razonamiento estratégico e incluso oportunista que deja entrever la gran capacidad de 
Brown para obtener beneficios de cualquier circunstancia por mínima que sea. El razonamiento prototípico del entrenador Brown no es moral ni mucho menos conforme a la ética de la virtud que proponen nuestros autores. Se trata más bien de un razonamiento estratégico, pues utiliza una lógica estratégica, pero no implica ni coherencia, dado que sólo tiene en cuenta el presente de su actuación sin contar con el pasado, ni con el futuro y no tiene en cuenta los modelos de excelencia ni la autoridad de los mismos que le llevan a estar en consonancia con una tradición deportiva que posee una historia. Simplemente se dedica a introducir una reforma para obtener mayores beneficios hacia el rendimiento de sus entrenados y que redunda en beneficio propio. Desde la ética de la virtud debe entenderse que la unidad de la vida moral es la unidad de un relato de búsqueda del concepto de lo bueno, desde donde poder ordenar los demás bienes existentes. Ello debe permitirnos ampliar nuestra comprensión del propósito y contenido de las virtudes y que nos permita entender el lugar de la interioridad y la constancia en la vida como búsqueda de lo bueno (MacIntyre, 2001: 270).

\section{Moral y valores morales en el deporte}

Lumpkin, Stoll y Beller (1994: 1-12), establecen una definición de moral referida específicamente a los motivos, acciones e intenciones que mantiene un sujeto moral en relación con los demás, mientras que la ética se refiere al estudio de la moralidad. Aplicada esta definición al mundo deportivo, la ética estudiará las cuestiones subyacentes, mientras que la moral se referirá a las acciones, motivos e intenciones de los participantes en el deporte. La relación con el otro será el factor determinante del valor moral en deporte, puesto que lo importante no es mí bien en el transcurso de la práctica deportiva, sino el bien que nos beneficia a todos. Lo importante es descubrir, desde el punto de vista moral, qué tiene mayor importancia en la práctica deportiva o, dicho con otras palabras, cuál es la finalidad del deporte. La confusión se produce entre aquellos que creen que lo más importante en deporte es ganar a toda costa, llegando a sostener que es lícito recurrir a todo tipo de estratagemas con la finalidad de obtener ventaja ante el oponente pasando por delante y proclamándose campeón. Pero también están aquellos que, por el contrario, consideran que en el deporte existen otro tipo de finalidades que la de ganar a toda costa y recurrir a todo tipo de argucias para conseguir esta meta.

Para evitar este tipo de confusiones nuestros autores establecen la distinción entre valor moral e inmoral en deporte, que no es más que una 
analogía, con la distinción de bienes internos o bienes externos a la práctica que podemos encontrar en la obra de MacIntyre. Éstos piensan que lo más importante son los bienes internos o valores morales en el deporte, que indiquen que lo más valioso de éste es competir bien, antes que proclamarse ganadores. Dichos valores permitirán al deporte mantener su estatus y su valor en sí, por encima de las tendencias egoístas. Por definición los bienes externos o valores inmorales, son contingentes y no tienen que ver con la auténtica finalidad moral de las prácticas, se referirían a todos aquellos deseos egoístas, tales como la obtención del honor, la ganancia económica, el prestigio y similares. Los bienes externos si se logran siempre son posesión y propiedad de un sujeto, lo que trae como consecuencia que cuantos más bienes externos obtenga alguien, menos habrá para el resto de miembros de una comunidad o de un equipo. Por ello, afirma MacIntyre (2001: 237) que los bienes externos son típicamente objeto de una competencia en la que debe haber perdedores y ganadores. Por el contrario, los bienes internos lo son por que únicamente se encuentran en el interior de una práctica y porque sólo pueden identificarse y reconocerse participando en la práctica en cuestión. Los bienes internos son el resultado de competir en excelencia, por ello su logro no beneficia a uno más que a los otros, donde lo que gana uno no lo pierden los otros. Por esta razón los bienes internos suponen un bien para toda la comunidad que participa en la práctica deportiva o cualquier otra.

Nuestros autores no pecan de ingenuos, pues saben muy bien que uno de los principales problemas de EEUU es que el capitalismo instaurado y su modelo socio-político favorece más a quienes valoren el éxito personal o grupal, incluso en deporte. Desde dicha tendencia se considera que todo aquel que no piense de la misma manera es una persona ingenua y poco realista, condenada al fracaso y al ostracismo (Lumpkin, Stoll \& Beller, 1994: 12). Pero no encontramos en estos autores una crítica enérgica frente a este reduccionismo social que supone la implantación o contaminación de los valores capitalistas, imperantes en una sociedad como la estadounidense, referidos al deporte. Dicha tendencia se hace presente en la práctica deportiva y le hacen perder su valor intrínseco haciéndola prevalecer a cualquier tipo de instrumentalización a favor de bienes, motivaciones y finalidades extrínsecas y dicho reduccionismo también será criticado por otros representantes de la ética del deporte como Robert. L. Simon (2006).

Lumpkin, Stoll y Beller apuestan por los valores morales en el deporte, que no se conciben como algo inútil y estúpido, sino como algo absolutamente valioso si queremos que el deporte no pierda su verdadero significado. Por esta razón, creen necesario dividir la 
aplicación de valores morales en tres fases: en primer lugar, se requiere del valor de saber actuar correctamente en el terreno de la competición deportiva; en segundo lugar, el valor de saber trabajar en equipo; y en tercer lugar, el valor de saber tomar decisiones que no perjudiquen a tu equipo ni a tu oponente. Dichos valores morales equivalentes a los bienes internos a la práctica, no sólo benefician a una parte, sino al todo, son en sí mismos valiosos y nos permiten alcanzar los modelos de excelencia moral que debe perseguir toda práctica que pretenda ser ética. Como acertadamente señala Donati (2011: 249), cuando las personas deciden algo como relación no deben pensar «esta relación tiene buenas consecuencias para mí», sino más bien, «esta relación es mi parte en una relación entre nosotros y tiene una buena consecuencia para todos».

Estos valores morales o bienes internos a la práctica deportiva, no pueden lograrse si antes no seguimos unas virtudes como guías morales que nos ayuden a ello. Recordemos que MacIntyre definía virtud como aquella cualidad humana adquirida y no impuesta, cuya posesión y ejercicio tiende a hacernos capaces de lograr los bienes internos. Para nuestros autores deben darse también un conjunto de virtudes morales que son justicia, honestidad, responsabilidad y beneficencia gracias a las cuales podamos obtener el valor moral de la práctica deportiva. La razón resulta evidente, sin el ejercicio de la virtud no se puede obtener el valor moral de la práctica deportiva ni el bien común. Especialmente en el caso del deporte moderno muy vinculado a la institución deportiva que puede propiciar la corrupción de las prácticas si no se cuenta con el horizonte de la virtud. Cuando hablamos de práctica debemos vincularla siempre a una institución que le permite subsistir en el tiempo, en nuestro caso la institución deportiva que goza de un amplio reconocimiento y estima en nuestras sociedades. El problema de las instituciones es que están típica y necesariamente comprometidas con los bienes externos (MacIntyre, 2001: 241). Esto puede apreciarse de manera ejemplar en la institución deportiva, que se caracteriza por utilizar el deporte para obtener el mayor rendimiento económico que redunde en mayores beneficios para los accionistas. De ahí la imperiosa necesidad de cultivar una serie de virtudes sin las cuales una práctica no podría resistir el poder corruptor de las instituciones. De este modo no prima en la práctica del deporte la obtención de recompensas, bienes y finalidades extrínsecas como la fama, el dinero o el prestigio mediático, como pretende la institución deportiva, sino todo lo contrario. Lo importante es que en la práctica deportiva, el «gozo de la actividad» y el «gozo del logro», no son los fines a los que tiende el agente moral. Ello se debe a que, como indica también MacIntyre (2001: 245), el gozo procede del éxito de la actividad de modo que la actividad lograda y la actividad gozada, son uno y el mismo estado. 
Consecuentemente lo característico de la virtud, si queremos que sea eficaz y nos ayude a conseguir los bienes internos, deberá ejercerse sin reparar en sus consecuencias. Pues, como ya dijo el Estagirita, la buena actuación, la actuación conforme a la virtud, es un fin. Pasemos a ver el listado de virtudes que nuestros autores proponen para hacer de la práctica deportiva una práctica ética.

\section{Justicia, honestidad, responsabilidad y beneficencia como principales virtudes morales en el deporte}

Cuando hablamos de virtud debemos partir de una definición provisional que entiende por ésta una cualidad humana adquirida cuya posesión y ejercicio tiende a hacernos capaces de adquirir los bienes internos o valores morales a la práctica. Lumpkin, Stoll y Beller (1994: 13) apuestan por cuatro virtudes morales que tienen un carácter interno, subjetivo e inconmensurable que las hace generalmente estimadas, ya que sin ellas las relaciones humanas dentro de la práctica deportiva resultarían muy difíciles y hostiles y no tendremos acceso a los bienes internos o valores morales. Podemos decir que estas virtudes tienen un valor inherente que las hace especialmente estimables al margen de su utilidad y al margen de su instrumentalización para cualquier otro fin extrínseco. Dichas virtudes poseen un atractivo universal porque son imprescindibles para el buen funcionamiento de las relaciones humanas, pues sin moral estas relaciones podrían resultar perjudicadas (Lumpkin, Stoll \& Beller, 1994: 25). Ello se debe a que las virtudes vienen a ser los bienes por referencia a los cuales definimos nuestra relación con los demás, con quienes compartimos los mismos modelos de excelencia y los mismos propósitos en la práctica (MacIntyre, 2001: 238). En efecto, como ha señalado acertadamente Jesús Conill (2006: 278), por encima de los intereses en juego, como el honor la fama y los beneficios lucrativos, debe imperar el principio de la vida de cada uno de los participantes, así como el valor mismo de la práctica (del deporte) como valor en sí.

Estas virtudes se consideraran el pilar central sobre el que se sustenta la práctica ética en el deporte y cuyo origen está en el núcleo de las guías morales por excelencia que ha tenido la humanidad en los últimos siglos como son la Biblia y el Corán, por lo que nuestros autores también dan importancia a la tradición. El peso de la tradición es importante en el desarrollo lógico de la virtud y constituye una de sus tres fases como ya había establecido MacIntyre. En efecto, toda práctica además de perseguir los bienes internos a los que sólo llegamos gracias al ejercicio de la virtud, conlleva modelos de excelencia y de obediencia a reglas. 
Entrar en una práctica supone aceptar la autoridad de dichos modelos y la cortedad de nuestra actuación. Pero toda práctica se da dentro de una determinada tradición. En relación con las virtudes la dimensión histórica es fundamental. Entrar en una práctica es entrar en relación no sólo con sus practicantes contemporáneos, sino con aquellos que nos han precedido.

Se trata de unas virtudes morales que se presentan como «principios universales» y que actúan como guías diciéndonos qué tipo de acciones, intenciones y motivaciones son obligatorias o cuáles deben estar prohibidas en la práctica deportiva. No obstante, pienso que es un grave error cimentar la ética en unos valores morales que se erigen como «principios universales» y que en el fondo se convierten en un «argumento de autoridad». En efecto, puede construirse una ética del deporte como ética de la virtud, ya que el comportamiento virtuoso, no sólo en deporte, sino en cualquier otro tipo de práctica, es moralmente necesario y positivo. Ahora bien, cimentar una ética del deporte en un listado de virtudes, que provienen de una determinada tradición, plantea de entrada ciertos inconvenientes o, al menos, ciertas inquietudes. En primer lugar, podría conducirnos al argumento de autoridad, pero también nos hace olvidar el valor del deontologismo ético en deporte. Tendríamos que ver cómo arbitrar el peso de las diversas tradiciones y cómo aplicarlo en los diversos contextos sociales. La práctica deportiva es una práctica intercultural, no multicultural, debido a que por encima de las diferencias culturales, debe imponerse una caracterización universal que valga para cualquier cultura, y que parta siempre de lo establecido por el Comité Olímpico Internacional (Donati, 2010).

En este contexto, como dicen Butcher y Schneider, la propuesta de estos autores no ofrece una fundamentación filosófica, ni un método defendible de decidir qué características o acciones debe caer en las definiciones relevantes así como tampoco ofrece un método para arbitrar entre pretensiones enfrentadas (Pérez Triviño, 2011: 27). Pienso que la correcta fundamentación de la moral no puede cimentarse solo en unas virtudes morales, se debe buscar la ayuda de otras categorías morales como las de «deber», «responsabilidad», o «compromiso» que en esta propuesta pasan a un estado secundario o subordinado.

En efecto, podemos hablar de un tipo u otro de virtudes morales en relación al fin que se persigue en deporte, por ello Lumpkin, Stoll y Beller, recopilan cinco posibilidades de utilizar estas virtudes en el deporte según el fin que se persiga: 1) si el fin está orientado hacia los resultados, entonces puedo decir que cualquier comportamiento es aceptable siempre que gane yo; 2) si existe una "ética» de ganar por encima de todo; 3) ganar es aceptable siempre que sea dentro de cierta 
normatividad; 4) ganar o perder son dos posibilidades viables en la competición, por ello lo importante es competir siempre dentro del espíritu del deporte siguiendo las reglas al pie de la letra; y 5) ganar o perder no es importante, pues la clave reside en jugar bien.

La aplicación de las cuatro virtudes que se dan en la práctica deportiva se produce en tres fases, que son: en primer lugar, se requiere del valor de saber actuar correctamente en el terreno de la competición deportiva; en segundo lugar, el valor de saber trabajar en equipo; y en tercer lugar, el valor de saber tomar decisiones que no perjudiquen a tu equipo ni a tu oponente.

A continuación me centraré en las cuatro virtudes que proponen nuestros autores.

1) La primera virtud es la justicia, coincidiendo así con MacIntyre (2001: 239), quien entendía que esta imprescindible virtud debe referirse a cómo tratamos a los demás en lo que respecta al mérito y merecimientos con arreglo a normas uniformes e impersonales, de forma que si nos apartamos de la norma de justicia en un caso particular marcaríamos nuestra relación con la persona en cuestión de manera diferenciada y especial. En el caso de Lumpkin, Stoll y Beller, distinguen cuatro tipos generales de justicia que son: la distributiva, la del procedimiento, la retributiva y la compensatoria. Cada uno de los cuatro tipos es inherente a la decisión y razonamiento moral aplicado al deporte.

La justicia distributiva se refiere a la percepción de la equidad y a la correcta distribución entre beneficios y cargas en aras a los beneficios obtenidos. Obviamente no conciben la justicia como equidad en términos rawlsianos, que se refiere a los dos principios de la justicia expuestos por John Rawls y que comprendía la igualdad de derechos ante la ley y la igualdad de oportunidades de los ciudadanos para mejorar su condición socio-económica. En este caso se refiere a una especie de igualdad entre los beneficios y cargas que deben ser igualmente llevados por todos los participantes sin excepción. Pero también coincide con la visión de MacIntyre y la de Aristóteles cuando hablaba de la justicia parcial que es la encargada de la distribución de honores (Ética a Nicómaco, V, 1131a).

La justicia en el procedimiento que se refiere a la equidad en las políticas de acción, de los procedimientos empleados y de los acuerdos utilizados para la obtención de los resultados. Se trata de que en todos los casos que surgen en la competición se apliquen los mismos procedimientos y acuerdos sin ninguna distinción, sea para favorecer a alguien o, por el contrario, para causarle un perjuicio. 
La justicia retributiva implica la percepción imparcial en relación a una infracción cometida en la prueba deportiva, con el propósito de detectar a un infractor o malhechor y de que su acción no quede impune ante los ojos de los demás participantes en el encuentro deportivo.

$\mathrm{Y}$, finalmente, encontramos la justicia compensatoria, que implica la percepción de la equidad para hacer el bien en el caso de que se haya cometido un daño hacia alguno de los participantes del encuentro. Se trata de una forma ya no de denunciar la imparcialidad que pueda mostrarse a través de una infracción, sino de compensar al ofendido por la falta que alguien puede haber cometido mientras competía, para que de esta manera el ofendido se vea compensado ante su ofensa.

2) La segunda virtud moral para el deporte es el de la honestidad, que es sin lugar a dudas uno de los valores más difíciles de conseguir tanto en la práctica deportiva como en cualquier otra práctica, pues, como decía Ortega y Gasset, abundan los «falsos ejemplares» que lejos de generar un comportamiento honesto, se dedican a dar a la esterilidad de sus acciones un valor positivo, aunque también señalaba Ortega que la perfección moral es una cualidad deportiva, algo que se añade a lo necesario e imprescindible (Ortega y Gasset, 1969: 99).

En efecto, el valor de la honestidad se basa en la premisa de que el actor no debe mentir, ni engañar en el transcurso de la competición deportiva. También MacIntyre (2001: 240) había incluido esta virtud y la denomina en multitud de ocasiones con el nombre de veracidad, para indicar precisamente que esta virtud garantiza el ejercicio de la imparcialidad y la disposición a confiar en los juicios de aquellos cuyos méritos en las prácticas les confieren autoridad para juzgar, presuponiendo la imparcialidad y veracidad de dichos juicios. Para nuestros autores la persona honesta en deporte es aquella que después de aceptar las normas, cumple con ellos, pues los acepta como veraces sin dudar de su honestidad. Para llevar a cabo este fin no deberán mostrar falsos heroísmos ni hipocresías, demostrando cosas que no tienen que ver con uno mismo, ni con algo que no eres realmente. Esto implica que todo aquel que quiera participar en un encuentro deportivo tendrá que ser moralmente honesto consigo mismo si quiere que su actuación en el terreno deportivo sea moralmente buena, para lo que deberá utilizar el mismo grado de bondad que utilizaría en su vida diaria. Entienden la honestidad como la condición o la capacidad de mostrarse como alguien de confianza y veraz en relación con los demás. Aplicado al mundo deportivo se referiría a nuestros compañeros de equipo y sobre todo a nuestros oponentes. Obviamente esto dependerá de la forma en que vemos a nuestro oponente, si es un obstáculo que se interpone entre yo y 
mi deseo de alcanzar la victoria, o si más bien es alguien igual a mí dentro del marco de la competición deportiva. Para ellos tan sólo debemos mostrarnos como alguien de confianza que no ataca por traición y que juega bien sus fichas, cumpliendo el reglamento y prescindiendo de trampas. Lo que sí reconocen que éste es el valor moral que más les cuesta cumplir a los deportistas de competición.

3) La tercera virtud moral es la responsabilidad, que ellos no entienden en el mismo sentido que lo hacen las nuevas corrientes neokantianas de ética, como son las éticas del discurso que se caracterizan por ser éticas de la responsabilidad. La ética del discurso, como la que proponen Jürgen Habermas y Karl-Otto Apel, se diferencia del deontologismo kantiano, en que tiene en cuenta la responsabilidad para que los agentes morales carguen con las consecuencias y subconsecuencias de las acciones morales que ellos mismos han consensuado. En el caso de Lumpkin, Stoll y Beller no se tiene muy en cuenta con asumir estas consecuencias, pues su idea de razonamiento moral se caracteriza porque es un tipo de razonamiento lógico, coherente e imparcial, pero olvida la importancia de que también sea consecuente y responsable ante las consecuencias de los agentes morales implicados en el deporte. Entienden que la responsabilidad aplicada al mundo deportivo se referirá a la rendición de cuentas por el comportamiento llevado a cabo por los participantes ante nuestros compañeros de equipo, ante nuestro entrenador y ante el juego mismo. Pero es mucho más complejo exigir cuentas cuando la moral viene desde fuera aprobada en virtud de la tradición o del argumento de autoridad, en lugar de haber sido uno mismo el que la tomase dialogando intersubjetivamente con el resto de afectados. Para ello recurren a la propuesta de Frankena (1973) que divide la noción de responsabilidad en tres aspectos: primero, la que se refiere a la responsabilidad ante nuestro carácter del que respondemos nosotros mismos; segundo, la responsabilidad ante las acciones que hemos cometido en el pasado; y tercero, la responsabilidad ante las acciones cometidas en el futuro. Pero no se tiene en cuenta la posibilidad de asumir las consecuencias a escala mundial de nuestras acciones, no sólo en el pasado o el futuro, sino también en el presente.

4) Por último, encontramos la beneficencia, que se entiende como la condición de no dañar, de prevenir los posibles daños y de hacer el bien en general. En sentido amplio e intencional aplicado al mundo deportivo, la beneficencia se entendería como un acto de cortesía común o de camaradería entre compañeros. Para mostrar a qué se refieren, nuestros autores se remiten al ejemplo del italiano Carlo Monti, quien en los 
Juegos Olímpicos de 1952 en un verdadero gesto de generosidad y benevolencia, ofreció el freno de su coche a su oponente cuando su freno quedó inoperante, mostrando así su compasión ante el dolor del otro.

Una vez establecidas las cuatro virtudes morales a las que nos tiene que hacer llegar el razonamiento moral con el que hay que dotar al deporte, Lumpkin, Stoll y Beller consideran necesario dar un paso más allá, pues piensan que para que la acción de los participantes sea éticamente aceptable en la práctica deportiva no sólo basta con los valores morales, sino que conviene defender unos principios morales. El esquema, de arriba hacia abajo, sería el siguiente:

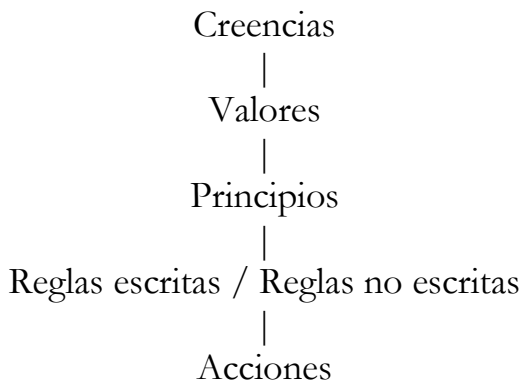

Este esquema diferirá del modelo aristotélico clásico de entender la acción de los agentes morales. Recordemos que para Aristóteles el hombre es el principio de la acción, pues en él confluyen el deseo, la deliberación que se refiere a los medios que nos conducen al fin y la elección entendida como un deseo deliberado entre aquellos medios que nos permiten acceder al fin natural: la eudaimonia. De la elección entre los vicios y las virtudes dependerá que alcancemos dicho fin. De este modo, entendía el Estagirita que la virtud es una disposición selectiva basada en el término medio, pero también la virtud es relativa a la elección, a la deliberación y a los deseos, pues el hombre es en definitiva inteligencia deseosa y deseo inteligente (Ética a Nicómaco, II, 1106a-1106b). Moncho aclara al respecto que una importante tesis de Aristóteles es que su concepto de virtud supone una armonía psíquica entre el deseo y la razón (Moncho, 2002: 165).

Ahora bien, la argumentación de Lumpkin, Stoll y Beller requiere dar un paso más allá, viendo el papel que desempeñan en el deporte tanto las reglas, sin las cuales no pueden darse las prácticas virtuosas ni alcanzarse los bienes internos a ellas, y los modelos de excelencia. Pero también requiere ver qué entienden por principio, junto con el concepto de ley 
moral, pues, como señala MacIntyre (2001: 248), cualquier moral de las virtudes requiere como contrapartida un concepto de ley moral.

\section{Reglas, principios, ley moral}

Recordemos cómo toda práctica que pretenda ser virtuosa conlleva además de bienes internos y modelos de excelencia la obediencia a reglas. Esto supone aceptar la autoridad de dichos modelos y la cortedad de nuestra propia actuación pero siempre desde el horizonte de las reglas (MacIntyre, 2001: 236). Es precisamente la falta de reflexión en torno a la naturaleza de las normas o reglas lo que más sorprende a MacIntyre en relación a la ética de Aristóteles. El autor de After virtue señala de manera crítica cómo la omisión más obvia y asombrosa del pensamiento del Estagirita es precisamente que las normas apenas son mencionadas en algún que otro pasaje de su Ética a Nicómaco. Pero, además, el Estagirita sostiene que la parte de la moral relacionada con la obediencia a normas, se refiere a la obediencia a las normas proclamadas por la ciudad-estado, que debe ser la encargada de sancionarlas como debe.

En la actualidad debemos a Rawls (1974: 211) la distinción entre dos conceptos de reglas: como mira sumaria, según la cual cada persona decide qué ha de hacer en los casos particulares, y como concepción de la práctica, en virtud de la cual se piensa que son las reglas las que definen la práctica y consecuentemente violar dichas reglas supone destruir la naturaleza misma de la práctica en cuestión. Pero también debemos a Rawls (1974: 210) la importancia de distinguir entre justificar una práctica, que él entiende como una especie de tecnicismo que se refiere a cualquier forma de actividad específica por un sistema de reglas, y la acción particular que cae dentro de ésta.

En el caso de Fraleigh (1984), representante del formalismo ético en deporte, la concepción de reglas que encontrábamos, era la de reglas como definitorias de la práctica deportiva, siendo éstas las que constituyen la naturaleza misma del deporte y las que marcan la zona de acción donde debe transcurrir la práctica de cualquier participante. Desde esta concepción de reglas se desestimaba todo tipo de trampa o violación, fueran de carácter intencional, no intencional o estratégica, pues se entiende que vulnera o destruye la naturaleza misma de la práctica deportiva al destruir su pilar fundamental: las reglas constitutivas. En el caso de Lumpkin, Stoll y Beller no son las reglas las que definen o caracterizan la práctica deportiva, pues este papel lo ocupan las virtudes que ellos proponen y que serían el resultado del razonamiento moral aplicado al deporte. Pese a ello, como buenos 
partidarios de una propuesta de ética de las virtudes marcadamente neoaristotélica à la MacIntyre, también tienen en cuenta en su propuesta la necesidad de seguir las reglas del deporte. Ello se debe a que además de las virtudes, en tanto que cualidades necesarias para obtener los bienes internos a la práctica y a la búsqueda de los modelos de excelencia, debe darse la dimensión de las reglas si queremos obtener dichos bienes y aspirar a los modelos de excelencia y al bien de una vida completa siempre dentro de una tradición social vigente. En este contexto, nuestros autores de nuevo olvidan profundizar en relación a la importancia de la naturaleza de dichas reglas y de nociones que deberían ir a la par de este importante concepto como son las de deber, obligación, responsabilidad, procedimiento o compromiso universalizable, como sí tiene en cuenta el deontologismo ético neokantiano.

Otra importante noción que cae bajo su consideración es la de principio. Entienden por «principio» técnicamente como una declaración escrita de todas las reglas elaboradas (Lumpkin, Stoll \& Beller, 1994: 28). Debe presuponerse que dichas reglas son veraces e imparciales y por ende nadie puede vulnerarlas en beneficio propio y en contra de los demás. El primer principio es el de lo justo, que se refiere a que tratemos a todas las personas con el mismo conjunto de reglas mediante el empleo de la distribución, el proceso adecuado, la retribución y la compensación. Este principio se muestra como una medida de equidad. El segundo principio consiste en ser honesto, el que se enmarca dentro de tres posibles conceptos negativos. Aquel que dice que mentir es verdaderamente deshonesto, el que dice que hacer trampas también es deshonesto, y finalmente el que desestima el robar, pues no tiene que ver con la honestidad. El tercer principio es el de lo responsable, que significa que tenemos que tomar conocimientos de nuestras acciones en el terreno deportivo y saber hacer lo que se tenía que hacer en ese momento, aceptando tanto las alabanzas como la culpa de lo que se haya hecho. El cuarto principio es el de la beneficencia, que cuenta con cuatro sub-apartados, que son: no hacer daño, eliminar los daños, prevenir los posibles daños, y finalmente hacer el bien. Este principio - que es casi idéntico al principio de no maleficencia y de beneficencia que se recoge en el Informe Beltmont como dos principios de la bioética- se erige para Lumpkin, Stoll y Beller como el emblema de la civilización y del sujeto civilizado que debe caracterizarse por no ser descortés.

Con todo, en el mundo del deporte sólo se aplican tres de los cuatro principios enumerados que son: el de lo justo, por medio del cual se debe conseguir que todos los participantes sean tratados equitativamente; el de la responsabilidad en virtud del cual se pretende que cada uno sea responsable ante cualquier situación que se le presente, aunque sería 
mejor haber dicho ser prudente; y finalmente el de la beneficencia, que hace que todo aquel que participe en la práctica sea civilizado, especialmente en relación a los terceros. Pero no se trata de asumir sólo la responsabilidad en solitario, sino en equipo siendo corresponsales, ya que el deporte, la mayoría de veces, se practica en equipo, se trata de saber cooperar, de tener metas comunes, sin olvidar que es conveniente aprender a cooperar para vivir y vivir bien, también en deporte, pero no como una exigencia moral categórica, sino como un consejo de prudencia (Cortina, 2011a: 126).

Otra obviedad en su propuesta es que toda moral de las virtudes, tal y como propone MacIntyre (2001: 248), requiere de un concepto de ley moral. Según MacIntyre (2001: 192), debe darse un vínculo clave entre las virtudes y la ley moral. La razón reside en que, saber cómo aplicar la ley moral sólo es posible para quien posee la virtud de la justicia. Ser justo equivale a dar a cada uno lo que merece, lo que requiere de criterios racionales de mérito y de que exista un acuerdo social sobre cuáles deben ser esos criterios. Esto resulta clave en el ámbito deportivo, pues además también Lumpkin, Stoll y Beller incluyen la virtud de la justicia en su listado de virtudes $y$, sin embargo, no tienen en cuenta el vínculo que debe darse entre la virtud y la ley moral. Especialmente si queremos aplicar la virtud de la justicia en la correcta distribución entre beneficios, en una política de acción que vele por la aplicación de los mismos procedimientos sin partidismos, en la correcta retribución ante las infracciones y en la compensación al equipo ofendido, tal y como ellos conciben la virtud de la justicia.

Tampoco tienen en cuenta la posibilidad de casos excepcionales y limítrofes dentro de la práctica deportiva y en donde, como afirmaba Rawls (1974: 241-242), será muy difícil la aplicación de cualquier concepción de regla. De lo que se impone la necesidad de la virtud de la phrónesis, que sí tiene en cuenta tanto la ética aristotélica como el propio MacIntyre, que alude a ella en relación al Estagirita y la vincula al juicio, que tiene un importante papel en la vida del hombre virtuoso. El hombre prudente, como señala acertadamente Michelakis (1961: 7), en tanto que personificación de la phrónesis, es el que sabe cómo actuar en cada caso particular, pero no basándose exclusivamente en lo que marcan las reglas, pues puede darse la posibilidad de casos donde sea muy difícil aplicarlas. 


\section{Violencia y deporte}

Lumpkin, Stoll y Beller también se hacen eco de uno de los principales problemas que por desgracia salpican cada vez más al mundo deportivo, me refiero a la violencia. Ésta es cada vez más presente en los acontecimientos deportivos, lo que quizás sea reflejo del «analfabetismo emocional» que sacude con mayor fuerza y frecuencia a nuestras sociedades, y que, como señala Cortina, se traduce como una nueva fuente de conductas agresivas, antisociales y antipersonales que se manifiestan tanto en la escuela, la familia y también el deporte (Cortina, 2007: 250).

La violencia en el deporte es la consecuencia más evidente de la falta de reflexión moral en torno a esta práctica y ha acabado convirtiéndose en tan omnipresente que algunos deportistas se muestran indiferentes cuando su oponente es lesionado o, peor aún, recurren a la violencia física con la intención de lesionar a su oponente y ganar una ventaja ilícita que les acerque a la victoria (Lumpkin, Stoll \& Beller, 1994: 63). La violencia en el deporte ha ido aumentando en las últimas décadas reflejando que los valores morales de la sociedad han cambiado, lo que ha producido la aparición de nuevas manifestaciones de violencia en el ámbito deportivo. De ahí que muchos autores como Jorge Olimpio Bento (2006; 2009; y Bento, Tani \& Prista, 2010) señalen que en Occidente, el deporte puede ocupar ese vacío moral y proporcionar una orientación axiológica a los individuos, teniendo en cuenta el declive y la falta de valores morales en nuestras sociedades.

En todo caso, la generalización del uso de la violencia en el deporte ha hecho que en muchas ocasiones los propios deportistas, que por desgracia están, poco o nada, familiarizados con la ética y la reflexión moral dentro del deporte, recurran al uso de la violencia para obtener una ventaja táctica. Éstos argumentan a su favor que dado que todos recurren al uso de la violencia se ven obligados ellos también a su empleo, pues de no hacerlo acabarían recibiendo golpes. Este tipo de razonamientos y comportamientos - peligrosamente relativistas- que justifican el uso indiscriminado de la violencia en el deporte, ha conducido a que muchos opositores vean a sus oponentes como objetos y no como personas. En este sentido, les ha llevado a cosificarlos no reconociendo que son seres con igual dignidad y con igualdad de oportunidades en alcanzar la tan ansiada victoria, pues, como muy acertadamente dijo Kant (1992), en la formulación del imperativo categórico: «Trata a los demás tanto en tu persona como en la de cualquier otro, siempre como fin y no sólo como medio». 
El desconocimiento de las reglas constitutivas del deporte, junto con el desconocimiento del espíritu del deporte acompañado por la falta de reflexión ética de sus participantes, ha conducido a la cosificación del oponente y a considerarlo como un obstáculo o enemigo que hay que superar física, mental y emocionalmente. Todo ello para llegar a la victoria, olvidando que antes que cosa es persona, lo que ha provocado que proliferen las acciones violentas dentro del deporte.

Para hacer frente a la omnipresencia de la violencia en el deporte, Lumpkin, Stoll y Beller proponen una lista no exclusiva de imperativos categóricos. Según ellos, estos imperativos serían el cimiento de la base moral en deporte y cuya aplicación por parte de los competidores supondría la paulatina eliminación de los comportamientos violentos. Los imperativos se distribuyen en seis, y son los siguientes: 1) Un verdadero deportista compite sacando lo mejor de sus capacidades dentro de lo establecido por las reglas constitutivas y prescriptivas, pero también lo hace dentro del espíritu o êthos del deporte. Incluiría los valores de justicia, honestidad y responsabilidad. 2) Se debe tratar de ganar desde el respeto por las reglas y por el espíritu del deporte. Incluiría los valores de justicia y de responsabilidad. 3) Un oponente no es un enemigo, sino un digno deportista que debe ser tratado como todo el mundo desea que se le trate. Principio de responsabilidad. 4) La venganza nunca es aceptable con independencia de la injusticia o violencia de la acción inicial. Principio de beneficencia. 5) Los juegos no se reproducen con la pretensión de intimidar, pues el ideal de un deporte debe de ser la búsqueda común de la excelencia (moral y personal) a través del desafío. Principio de justicia y beneficencia. 6) La deportividad requiere modestia, humildad en la victoria y en los elogios, así como respeto en la derrota. Lo que requiere de una «pedagogía de la derrota», proyecto inacabado en la sociología del deporte de José María Cagigal (1972: 73).

\section{Conclusiones}

Llegados a este momento estamos en condiciones de extraer algunas conclusiones. En primer lugar, la propuesta de ética del deporte elaborada por Lumpkin, Stoll y Beller, debe entenderse como una forma de sustancia-lismo ético neoaristotélico, à la MacIntyre, pese a que en muchos aspectos todavía les falta ahondar más sobre el pensamiento del autor de After virtue. El sustancialismo ético, que podemos dividir en neohegeliano y neoaristotélico, se caracteriza por entender la moral desde conceptos tales como los de praxis, telos, virtud, comunidad y vida 
buena. Desde esta posición, dichos conceptos pasan a un primer plano de la reflexión ética, postergando a un lugar secundario o subordinado otras nociones morales como las de reglas, deberes, responsabilidad... etc. Esto puede apreciarse en la propuestas de ética del deporte como «bolsa de virtudes» que elaboran Lumpkin, Stoll y Beller, donde toda la reflexión ética gira en torno a este listado de virtudes, pero donde se entiende que lo importante es la búsqueda de los valores morales, frente a los inmorales, que en definitiva es la búsqueda de los bienes internos, frente a los externos, dentro de la práctica deportiva, gracias al ejercicio de la virtud. Siempre dentro del contexto de una determinada tradición, que en el caso de Lumpkin, Stoll y Beller serían las dos grandes guías morales de la humanidad: la Biblia y el Corán. Pero además dejando en un segundo plano la reflexión en torno a las reglas del deporte.

En segundo lugar, en esta propuesta ético-deportiva no sólo es evidente la falta de un método defendible, como denunciaban al respecto Butcher y Schneider, lo que no sólo demuestra la falta de una fundamentación filosófica en su propuesta ética, sino también otro importante problema que ya había sido indicado por el propio MacIntyre en After virtue. Se trata de que en la propuesta de Lumpkin Stoll y Beller, se ofrece una definición de virtud que es diferente a la que dan otras propuestas de ética del deporte como «bolsa de virtudes», pensemos en la que elaboran Brenda Bredemeir y David Shields en su libro titulado: Character Development and Physical Activity. Lumpkin, Stoll y Beller, nos ofrecen un listado de virtudes diferentes entre sí con otras propuestas similares, lo que dificulta saber qué virtudes aceptar para la elaboración de una ética del deporte. Además, otro problema que encontramos y que también había sido denunciado por Butcher y Schneider, es que en algunos casos las virtudes que estos autores proponen se contradicen con las reglas constitutivas de un deporte como ejemplificaba el caso del rugby, mostrando la imposibilidad y arbitraje ante pretensiones enfrentadas.

Pienso que si algo nos ha enseñado el procedimentalismo ético es que la cuestión fundamental de la filosofía práctica consiste en preguntarnos por los procedimientos y premisas desde donde las justificaciones pueden tener un poder generador de consenso. En el caso de la práctica deportiva, no se trata de buscar un consenso que permia legitimar qué tipo de reglas deben caracterizar a dicha práctica cada vez que iniciamos una competición deportiva, ya que es el Comité Olímpico Internacional quien las ha establecido previamente, pero sí puede ayudarnos a establecer qué normas morales deberán guiar nuestra conducta en el terreno deportivo según el contexto y nuestra facticidad. Aunque la ética se refuerza al incorporar virtudes, como puedan ser las 
que sostienen nuestros autores, se necesita algo más que virtudes para una propuesta de ética del deporte. Debemos leer los procedimientos en la praxis concreta y buscar la universalidad que posibilite una verdadera atmósfera ética, es necesario trascender los contextos concretos de manera crítica para luego volver a ellos. En este punto creo que también hemos de tener en cuenta la aportación de la hermenéutica crítica que nos ayuda a trascender los contextos concretos para ampliar nuestra visión. En efecto, la hermenéutica es crítica y la crítica ha de ser hermenéutica, como señala Conill (2006: 211), para lo que se hace necesario trascender los contextos, pues no se trata de quedarnos inmersos en ellos viendo meramente cuáles son las señales dominantes o las relaciones de poder. Debemos trascenderlos para poder volver a ellos en particular desde una perspectiva universal. Pero otra aportación de esta ética hermenéutica reside en que la razón misma no es pura, sino impura y necesita de teorías normativas que permitan poner en cuestión los juicios morales habituales (Cortina, 2011b: 220). La gran aportación de la ética hermenéutica crítica de la facticidad que propone Conill (2006: 160) siguiendo la aportación gadameriana y kantiana, nos debe ayudar a discernir - siempre desde un fondo normativo- un sentido del bien, pero en la situación concreta.

\section{REFERENCIAS}

-Bento, J. O. (2006). Do desporto como um projecto ético para uma mudanza das mentalidades e atitudes. Pedagógia do desporto. Rio de Janeiro: Editora Guanabara Koogan.

-Bento, J. O. (2009). Do corpo e do activismo na conjura de mercado e consumo. Revista portuguesa de ciencias do desporto, IX, 2-3.

-Bento, J. O., Tani, G; \& Prista, A. (2010). Desporto e Educação física em Português. Contributo para o XIII Congresso de Ciências do Desporto e de Educação Física dos Países de Língua Portuguesa (Maputo: 30 de Março - 2 de Abril de 2010). Porto: CIFI2D-Centro de Inovação, Formação, Investigação e Intervenção em Desporto, Faculdade de Desporto da Universidade do Porto.

-Butcher, R. \& Schneider, A. (2002). Fair play as respect for the game. Journal of the Philosophy of Sport, 25, 1-22.

-Cagigal, J. M. (1972). Deporte, pulso de nuestro tiempo. Madrid: Editora Nacional. -Conill, J. (2006). Ética bermenéutica. Madrid: Tecnos.

-Cortina, A. (2001). Ética sin moral. Madrid: Tecnos.

-Cortina, A. (2007). Ética de la razón cordial: Educar en la ciudadanía del siglo XXI.

Oviedo: Edición Novel. 
-Cortina, A. (2011a). Neuroética y neuropolítica: Sugerencias para la educación moral. Madrid: Tecnos.

-Cortina, A. (2011b). Neuroética: ¿ética fundamental o ética aplicada? Diálogo Filosófico, 80, 205-224.

-Donati, P. (2010). Oltre il multiculturalismo: La ragione relazionale per un mondo comune. Roma- Bari: Editori Laterza.

-Donati, P. (2011). Sociologia della riflessività: Come si entra nel dopo-moderno. Bologna: Il Moulino.

-Feezell, R. (1986). Sportsmanship. Journal for the Philosophy of Sport, 1, 1-13.

-Fraleigh, W. P. (1984). Right actions in Sport. Champaign IL: Human Kinetics publishers.

-Frankena, W. K. (1973). Ethics. Englewood Cliffs, NJ: Prentice Hall.

-Guttmann, A. (1978). From ritual to record: the nature of modern sport. New York: Columbia University Press.

-Guttmann, A. 2000). Development of Modern Sport. En J. Coakey \& E. Dunning (Eds.), Handbook of Sport Studies. London: Sage.

-Kant, I. (1992). Fundamentación de la metafísica de las costumbres. Madrid: Sociedad Económica Matritense de Amigos del País.

-Lumpkin, A. Stoll, S. \& Beller, J. (1994). Sport ethics: Applications of Fair Play. St Louis: MO-Mosby.

-MacIntyre, A. (2001). Tras la virtud. Barcelona: Crítica.

-Michelakis, E. (1961). Aristotle's theory of practical Principles. Atenas: Cleisiounis Press.

-Moncho, J. R. (1972). La unidad de la vida moral según Aristóteles. Valencia: Anales del Seminario de Valencia.

-Moncho, J. R. (2002). Ética de la virtud. La ciudad de Dios (San Lorenzo del Escorial), CCXV (1), 155-180.

-Ortega y Gasset, J. (1969). El espectador. Madrid: Salvat Editores.

-Pérez Triviño, J. L. (2011). Ética y deporte. Bilbao: Desclée de Brouwer.

-Rawls, J. (1974). Dos conceptos de reglas. En P. Foot, Teorías sobre la ética. México: Breviarios de F.C.E.

-Russo, G. (2011). La società della welness: Corpi sportivi al traguardo della salute. Milano: Franco Angeli.

-Simon, R. L. (2006). Fair Play: The Ethics of Sport. USA: Westview Press.

Sumario: 1. El horizonte filosófico de la virtud: De Homero a MacIntyre; 2. Valor y razonamiento moral en el deporte en A. Lumpkin, S. K. Stoll y J. M. Beller; 3. Moral y valores morales en el deporte; 4. Justicia, honestidad, responsabilidad y beneficencia como principales virtudes morales en el deporte; 5. Reglas, principios, ley moral; 6. Violencia y deporte; Conclusiones; Referencias. 